\title{
Research on simulation technology of wireless sensor in swimming mechanics
}

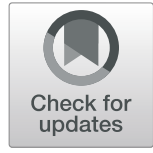

Zhiwen Xu

\author{
Correspondence: jijiurongyuan925@ \\ 163.com \\ Department of Physical Education, \\ Chang'an University, Xi'an 710064, \\ China
}

\begin{abstract}
In order to improve the quantitative teaching level of swimming sports, it is necessary to carry out quantitative analysis of swimming sports mechanics. A method of swimming mechanics analysis based on computer simulation technology is proposed. The ARMA model is used to analyze the mechanics of the limb movement of swimming, the Lagrange dynamics model is used to model the characteristics of the mechanics of swimming movement, and the distributed characteristic parameter model of the dynamics of swimming is analyzed in the space of six degrees of freedom. The dynamic distribution space of swimming motion is obtained by using the forward kinematics analysis model, and the inverse kinematics of multiple degrees of freedom is solved by analytic method, and the global and local joint force and force parameters in swimming are estimated. The model of swimming mechanics is realized. The simulation results show that the proposed method is accurate and accurate in estimating the mechanical parameters of swimming movement, and it can effectively guide swimming training.
\end{abstract}

Keywords: Computer simulation technology, Wireless sensor, Lagrange dynamic model, Human body spatial position model

\section{Introduction}

A swimming competition is mainly composed of five links: starting, route, turning, sprint, and edge technique. As the first step of the whole process, swimming and departure has great significance. With the improvement of the competitive level of swimming in the world and the increasing intensity of the competition, different water sensations have different effects on the swimming performance of the athletes. Only by improving the water sense of the swimmers can they become more relaxed in the process of swimming. In order to get a better result in swimming, in this study, there are two main methods of water sense practice, one is fist grip exercise and the other is using water stroke exercise. If a swimmer has mastered the key points of action, but he lacks a good sense of water, we can use the method of clenching to make athletes tighten their double fists, avoid palm contact with the current, and use their fists to feel the direction and strength of the current in the water. We should pay attention to the need to control the stroke strength of the fist well, not too large [1]. By limiting the number of strokes and using the palm of the hand to magnify the resistance

(c) The Author(s). 2020 Open Access This article is licensed under a Creative Commons Attribution 4.0 International License, which permits use, sharing, adaptation, distribution and reproduction in any medium or format, as long as you give appropriate credit to the original author(s) and the source, provide a link to the Creative Commons licence, and indicate if changes were made. The images or other third party material in this article are included in the article's Creative Commons licence, unless indicated otherwise in a credit line to the material. If material is not included in the article's Creative Commons licence and your intended use is not permitted by statutory regulation or exceeds the permitted use, you will need to obtain permission directly from the copyright holder. To view a copy of this licence, visit http://creativecommons.org/licenses/by/4.0/. 
encountered by the athletes during the stroke, the paddling method can enhance the athletes' feeling of water and facilitate the athletes to adjust the frequency and amplitude of their own stroke. Through the analysis of swimming movement mechanics, it is of great significance in improving the training level and studying the swimming sports mechanics analysis model to improve the swimming technical movement [2].

In order to realize the kinematic analysis of swimming, it is necessary to construct the dynamic model of the body and to analyze the dynamics of the body. From the point of view of modeling theory of lower limb and knee movement chain, the geometric control and behavior control mechanism of lower limb and upper limb movement chain in the course of useful swimming should be analyzed [3]. In reference [4], the kinetic energy decomposition and mechanical model of a moving arm with 7 rotational degrees of freedom are solved by geometric method. First, the CCD algorithm is used to make the inverse motion error between the virtual pose and the current position and pose. The method of combining mechanical structure with inverse kinematics of human body structure is used to decompose the characteristics of lower limbs and knees during swimming, and the global force is analyzed. In this way, it is impossible to estimate the local force mode of each joint of the limb, and the human motion model cannot be constructed accurately. The lower limbs and knees are decomposed with 6 degrees of freedom, and the dynamic mathematical modeling and mechanics are carried out. The composition of the algorithm is complex and the adaptability is not good [1].

Aiming to solve the above problems, this paper presents a mechanical analysis method of swimming motion based on computer simulation technology [3]. The ARMA model is used to analyze the mechanics of the limb movement of swimming, the Lagrange dynamics model is used to model the characteristics of the mechanics of swimming movement, and the distributed characteristic parameter model of the dynamics of swimming is analyzed in the space of six degrees of freedom. The dynamic distribution space of swimming motion is obtained by using the forward kinematics analysis model, and the inverse kinematics of multiple degrees of freedom is solved by analytic method, and the global and local joint force and force parameters in swimming are estimated. The model of swimming mechanics is realized. Finally, the performance test is carried out through the simulation experiment, which shows the superior performance of this method in improving the ability of swimming mechanics analysis.

\section{Methods}

\subsection{Structure of human sports chain in swimming}

In this paper, the Lagrange dynamic model is constructed, and the structure of swimming human body motion chain is analyzed in six degrees of freedom space combined with six degrees of freedom spatial impact dynamics model construction method. The kinetic energy of the sagittal knee impact force in swimming is decomposed and described, and the local mechanical parameters of the knee joint are solved [5]. The global analysis of the mechanical parameters in swimming process is carried out by using the 7-bar driving structure, and the optimal solution vector is obtained. Firstly, the spatial position model of swimming is analyzed, as shown in Fig. 1. 


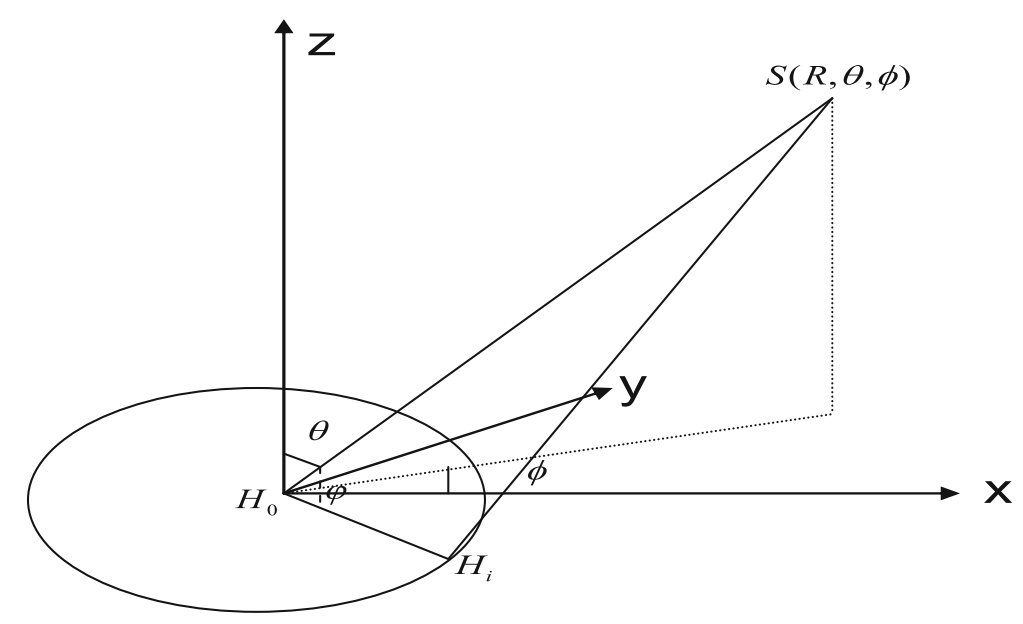

Fig. 1 Human body spatial position model during swimming

In Fig. 1, the swimming field is a standard swimming field and the amplitude of the space vector of the human body entering the water, the array spacing of the space position of swimming [6], the elevation angle of the human body entering the water, and the azimuth of the waist and the left (right) arm $\theta$ are correlated with the distance $S$. The estimated parameters of three rotational degrees of freedom are as follows:

$$
\frac{c \tau_{i}}{r}=\frac{r^{2}-c^{2} \tau_{i}^{2}}{2 R r}+\cos \phi_{i}(i=1, \quad 2 \ldots M)
$$

Because in swimming, the space position of entering water is a uniform linear array, according to motion planning theory, it can be proved that:

$$
\cos \left(\phi_{i}\right)+\cos \left(\phi_{i+M / 2}\right)=0
$$

The dynamic decomposition structure model of inverse kinematics of swimming athletes was obtained. In the initialization study of mechanical decomposition, $\sum_{i=1}^{M} \cos \phi_{i}$ is obtained. The azimuth distance $R$ of the position and posture of human body in the process of entering and leaving the water can be deduced as follows:

The joint at the palm of the world coordinate system construction, knee joint unit $i$ in the process of force, and the body into the water of the arm movement chain as:

$$
\begin{gathered}
{ }^{4} \mathbf{T}_{7}=\prod_{i=5}^{7-1} \mathbf{T}_{i}\left(q_{i}\right)=\prod_{i=4}^{1}{ }^{i-1} \mathbf{T}_{i}^{-1}\left(q_{i}\right) \cdot{ }^{0} \mathbf{T}_{4} \\
=\left[\begin{array}{cccc}
\mathbf{n}_{e} & \mathbf{o}_{e} & \mathbf{a}_{e} & \mathbf{p}_{e} \\
0 & 0 & 0 & 1
\end{array}\right]
\end{gathered}
$$

In that kinematic chain structure of the swimming exercise human body, the joint can be approximate as a fixed center [7], and the state equation of the motion chain of the human body in the swimming exercise is:

$$
m \frac{d V}{d t}=P \cos \alpha-X-m g \sin \theta
$$




$$
m V \frac{d \theta}{d t}=P \sin \alpha+Y-m g \cos \theta
$$

in which, $m$ is the mass of the force unit in swimming, $P$ is the moment of momentum of the right foot in the process of entering the water. $\theta$ is the rotation vector of the joints of the two limbs. It provides a model basis for kinematic mechanics analysis [8].

\subsection{Model analysis of swimming parameters}

On the basis of the above model construction and kinematic chain analysis, the kinematic parameter model of swimming is analyzed, and the kinematics of swimming is analyzed in six degrees of freedom, and the joint angle space is obtained by the forward kinematics algorithm. Thus, the kinetic energy of the body's functional parts in the water is obtained as follows:

$$
K=\frac{1}{2} \sum_{i=1}^{6}\left[I_{i} q_{i}^{2}+m_{i}\left(x_{i}^{2}+z_{i}^{2}\right)\right]
$$

The upper limb mass $m_{3}$ is regarded as a center of mass, and the inverse kinematics of multiple degrees of freedom is solved by analytic method. The potential energy of swimming athletes after entering the water is expressed as follows:

$$
P=\sum_{1=0}^{6} m_{i} g z_{i}
$$

Under the limited conditions, the equal-position equivalent kinematic chain structure of continuous swimming using Lagrange dynamic model is shown as follows:

$$
\int_{V} \varepsilon_{i j} d V=\int_{V} \varepsilon_{i j}^{*} d V
$$

The energy equivalent relationship between swimming and swimming is obtained as follows:

$$
\int_{V} \sigma_{i j} \varepsilon_{i j} d V=\int_{V}\left(\sigma_{i j}^{*} \varepsilon_{i j}^{*}\right) d v
$$

Under macroscopical conditions [9], the equivalent stress expression of twodimensional periodic structure for swimmers entering water is as follows:

$$
\sigma_{i j}^{*}=\frac{1}{V} \int_{V} \sigma_{i j} d V
$$

The reference coordinate system for swimming athletes to enter 7 degrees of freedom in the right arm:

$$
\sigma_{i j}{ }^{*}=C_{i j k l} \varepsilon^{*}{ }_{i l}
$$

In the water state, the joint vector of the elbow joint position of the right arm is calculated as: 


$$
\begin{aligned}
& C_{i j k l}=2 \frac{1}{V} W^{*} \overline{\varepsilon_{i j}} \frac{1}{\varepsilon^{2}{ }_{i j}} i=j, k=l, i=k \\
& C_{i j k l}=\frac{1}{2 V} W^{*} \overline{\varepsilon_{i j}} \frac{1}{\varepsilon^{2}{ }_{i j}} i \neq j, k \neq l, i=k, j=l \\
& C_{i j k l}=\frac{1}{4 V} \frac{\left(W^{*} \overline{\varepsilon_{i j} \varepsilon_{k l}}\right)-W^{*} \overline{\varepsilon_{i j}}-W^{*} \overline{\varepsilon_{k l}}}{\overline{\varepsilon_{i j} \varepsilon_{k l}}} i \neq j, k \neq l \\
& C_{i j k l}=\frac{1}{2 V} \frac{\left(W^{*} \overline{\varepsilon_{i j} \varepsilon_{k l}}\right)-W^{*} \overline{\varepsilon_{i j}}-W^{*} \overline{\varepsilon_{k l}}}{\overline{\varepsilon_{i j} \varepsilon_{k l}}} i=j, k \neq l
\end{aligned}
$$

The homogeneous coordinate transformation matrix of swimming motion can be obtained by the calculation of the previous modulus. Based on the analysis of swimming motion parameter model, the kinetic analysis of motion mechanics can be carried out [10].

\section{Model improvement design and implementation}

The ARMA model is used to analyze the mechanics of the limb movement of swimming, the Lagrange dynamics model is used to model the characteristics of the mechanics of swimming movement, and the distributed characteristic parameter model of the dynamics of swimming is analyzed in the space of six degrees of freedom [11]. Using the inverse kinematics analytical model, the total joint structure $z_{i}$ of swimming under six degrees of freedom is decomposed, and the force component is calculated as follows:

$$
\begin{aligned}
& z_{0}=0 \\
& z_{1}=a_{1} \cos q_{1} \\
& z_{2}=l_{1} \cos q_{1}+a_{2} \cos q_{2} \\
& z_{3}=l_{1} \cos q_{1}+l_{2} \cos q_{2}+a_{3} \cos q_{3} \\
& z_{4}=l_{1} \cos q_{1}+l_{2} \cos q_{2}+a_{4} \cos q_{4} \\
& z_{5}=l_{1} \cos q_{1}+l_{2} \cos q_{2}+l_{4} \cos q_{4}+a_{5} \cos q_{5} \\
& z_{6}=l_{1} \cos q_{1}+l_{2} \cos q_{2}+l_{4} \cos q_{4}+l_{5} \cos q_{5}+a_{6} \cos q_{6}
\end{aligned}
$$

Based on grid decomposition, the Lagrange dynamic model of swimming athletes with redundancy in the right arm in water is used for continuous mechanical decomposition [12]. The forward and reverse kinematics equations of swimming athletes entering the left arm are obtained as follows:

$$
\begin{aligned}
C_{i j k l} & =2 \frac{1}{V} W^{*} \overline{\varepsilon_{i j}} \frac{1}{\varepsilon^{2}{ }_{i j}} i=j, k=l, i=k \\
C_{i j k l} & =\frac{1}{2 V} W^{*} \overline{\varepsilon_{i j}} \frac{1}{\varepsilon^{2} i j} i \neq j, k \neq l, i=k, j=l \\
C_{i j k l} & =\frac{1}{4 V} \frac{\left(W^{*} \overline{\varepsilon_{i j} \varepsilon_{k l}}\right)-W^{*} \overline{\varepsilon_{i j}}-W^{*} \overline{\varepsilon_{k l}}}{\overline{\varepsilon_{i j} \varepsilon_{k l}}} i \neq j, k \neq l
\end{aligned}
$$




$$
C_{i j k l}=\frac{1}{2 V} \frac{\left(W^{*} \overline{\varepsilon_{i j} \varepsilon_{k l}}\right)-W^{*} \overline{\varepsilon_{i j}}-W^{*} \overline{\varepsilon_{k l}}}{\overline{\varepsilon_{i j} \varepsilon_{k l}}} i=j, k \neq l
$$

In which, $\boldsymbol{\xi} \in R^{10}$ is an arbitrary vector, $\mathbf{J}^{+} \mathbf{p}_{e}$ is the inverse kinematic solution of joint velocity, and the Jacobian matrix of swimming athletes entering the water $\operatorname{arm}$ is $\mathbf{J}(\boldsymbol{\theta}) \in$ $\mathbf{R}^{6 \times 10}$, the energy equivalent relation of motion burst is obtained as follows:

$$
\boldsymbol{\theta}=\mathbf{J}^{+} \mathbf{p}_{e}+\left(\mathbf{I}-\mathbf{J}^{+} \mathbf{J}\right) \boldsymbol{\xi}
$$

The swimming athletes enter the water waist in the three-dimensional space relative to the movement of the inertia reference system $\Sigma_{I}$ of the lower extremity. The Lagrange dynamics experiment of swimming is carried out [13], and the dynamic analysis model of the ankle joint is obtained as follows:

$$
M(q) q+K(q, q)+H(q)=T
$$

in which, $\mathbf{p}_{t}=\left[x_{t}, y_{t}, z_{t}\right]^{T}$, the relation between the translation constant $m_{L 3}$ and $m_{R 3}$ in the three directions of the upper formula is expressed as follows:

$$
\begin{aligned}
& x_{L 3}=l_{1} \sin \theta_{1}+l_{2} \sin \left(\theta_{1}-\theta_{2}\right)+\alpha_{3} \sin \left(\theta_{1}-\theta_{2}+\theta_{3}\right)-\alpha_{0} \\
& x_{R 3}=\alpha_{6}-l_{5} \sin \theta_{6}+l_{4} \sin \left(\theta_{5}-\theta_{6}\right)-\alpha_{3} \sin \left(\theta_{4}-\theta_{5}+\theta_{6}\right) \\
& \frac{m_{L 3}}{m_{R 3}}=\frac{x_{R 3}}{x_{L 3}} \\
& m_{3}=m_{L 3}+m_{R 3}
\end{aligned}
$$

According to the classical gradient method proposed by Liegeois, the prediction of swimming mechanics is carried out. The relationship between the predicted value and the real value is shown as follows:

$$
K=\frac{1}{2} \sum_{i=0}^{2}\left[I_{i} \dot{q}_{i}^{2}+m_{i}\left(\dot{x}_{i}^{2}+\dot{z}_{i}^{2}\right)+\frac{1}{2}\left(I_{L 3} \dot{q}_{3}^{2}+m_{L 3}\left(\dot{x}_{3}^{2}+\dot{z}_{3}^{2}\right)\right)\right]
$$

The distribution characteristic parameter model of swimming motion mechanics is analyzed in the six degrees of freedom space. The dynamic distribution space of swimming motion is obtained by using the forward kinematics analysis model, and the inverse kinematics solution of multiple degrees of freedom is realized by analytic method. The results of estimating the mechanical parameters of swimming are expressed as follows:

$$
u(t)=\left\{\begin{array}{cc}
u_{1}(t)=A(t) \exp \left\{j\left[2 \pi K \ln \left(1-\frac{t}{t_{0}}\right)\right]\right\} & -\frac{T}{2}<t<0 \\
u_{2}(t)=u_{1}^{*}(-t) & 0<t<\frac{T}{2}
\end{array}\right\}
$$

The estimated results of the inertial force characteristics of swimming are expressed as follows:

$$
\tau_{0}=\frac{1}{2}\left(b_{1}+b_{2}\right)
$$




$$
s_{0}=\left(a *+\frac{b_{1}-b_{2}}{2 t_{0}}\right)
$$

Above all, the distribution characteristic parameter model of swimming motion mechanics is analyzed in six degrees of freedom space, and the dynamic distribution space of swimming motion is obtained by using forward kinematics analysis model, which realizes the mechanical analysis and modeling of swimming movement.

\section{Experiment}

In order to test the application performance of this method in swimming mechanics analysis, the simulation experiment is carried out. The experiment is designed by Matlab 7. After the research object is determined, the experimental results are compared and analyzed by the contrast experiment method. The specific operation is to divide 10 swimmers into a control group and experimental group on average, and then to conduct a $200-\mathrm{m}$ backstroke competition, make good records, and then conduct training for 3 months. In the course of the experiment, the experimental group used the effective backstroke technique to train, while the control group adopted the normal training method. The two groups of swimmers maintained the same training time every day, and the other training contents and intensity remained unchanged. At the end of the experiment period, the two groups of swimmers were given 200-m backstroke, then the results of the two groups were compared, and the results of the two groups were compared with the results of backstroke before the experiment. Finally, the comparative results were observed to find out the gap between the control group and the experimental group, and to judge the effectiveness of backstroke technique. After three months of training, the average results of 200-m backstroke in the control group and the experimental group were compared with those before training, and the simulation results of the mechanical parameters of swimming movement were obtained as shown in Table 1 .

Based on the results in Table 1, a descriptive statistical analysis is carried out, and the results of mechanical simulation are shown in Fig. 2.

The results of the analysis show that the results of backstroke in the experimental group are obviously improved, and there is a great time difference between the experimental group and the control group. The average result of the backstroke in the control group is $2.1 \mathrm{~s}$ behind the average result of the experimental group, which shows that the effective backstroke technique is effective. It plays an important role in improving the technique of swimmers. Network transmission simulation test during swimming dynamics transmission is shown in Fig. 3. Network packet loss rate test during swimming dynamics transmission is shown in Fig. 4.

There is a significant difference between the two, and if $P<0.05$, there is statistical significance, and it is proved that improving the backstroke technology of swimmers can improve the backstroke results. It can also be seen from the chart that the backstroke scores of both the control group and the experimental group were significantly improved before and after training, in which the time used in the control group was reduced by $2.10 \mathrm{~s}$, and the time in the experimental group was reduced by $3.90 \mathrm{~s}$, which 
Table 1 Description of simulation results of swimming mechanics parameters

\begin{tabular}{llll}
\hline Collection point & Left arm $x(\mathrm{~m})$ & Right arm $y(\mathrm{~m})$ & Stress $(\mathrm{N})$ \\
\hline 1 & 2.543 & 3.232 & 34.45 \\
2 & 2.921 & 8.232 & 65.32 \\
3 & 8.242 & 6.241 & 29.32 \\
4 & 0.421 & 3.445 & 65.92 \\
5 & 8.624 & 6.544 & 6553 \\
6 & 6.725 & 6.323 & 76.23 \\
7 & 5.453 & 3.435 & 34.32 \\
8 & 7.765 & 5.968 & 65.32 \\
9 & 5.545 & 3.887 & 33.74 \\
10 & 6.675 & 5.897 & 14.24 \\
11 & 8.654 & 5.245 & 43.45 \\
12 & 6.764 & 8.345 & 32.35 \\
13 & 3.698 & 8.343 & 48.34 \\
14 & 9.878 & 5.464 & 32.22 \\
15 & 8.876 & 8.854 & 32.43 \\
16 & 8.577 & 5.567 & 32.35 \\
17 & 7.876 & 4.244 & 52.46 \\
18 & 2.676 & 8.567 & 58.87 \\
19 & 4.465 & 9.456 & 29.53 \\
20 & 9.378 & 2.343 & 36.41 \\
\hline
\end{tabular}
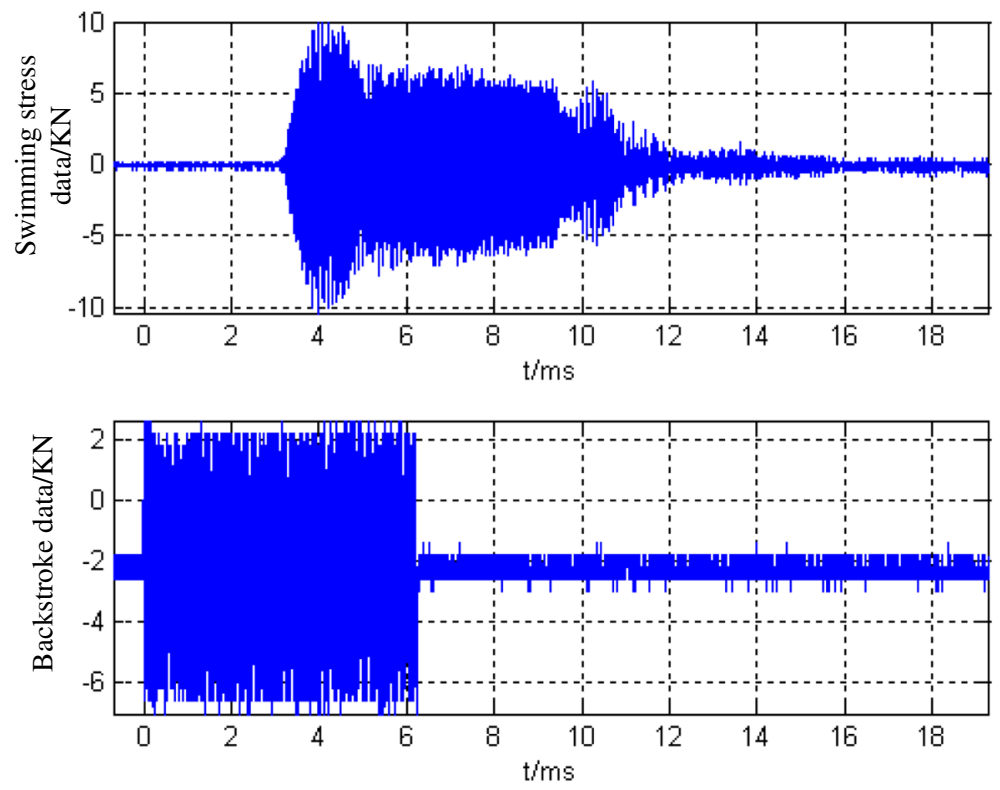

Fig. 2 Results of swimming mechanics simulation 


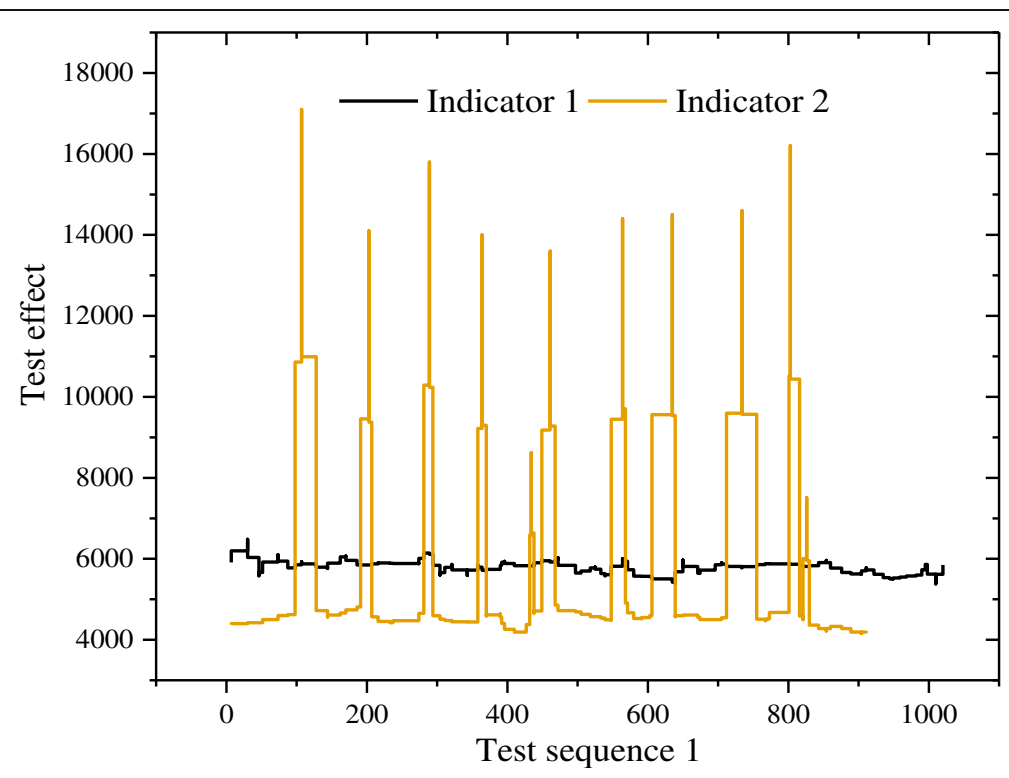

Fig. 3 Network transmission simulation test during swimming dynamics transmission

was more obvious than that in the control group. The accuracy of the estimation of swimming mechanical parameters by different methods is tested. The comparison results are shown in Fig. 5. The analysis shows that the accuracy of the method is higher than that of other methods.

\section{Results and discussion}

In this paper, a method of swimming mechanics analysis based on computer simulation technology is proposed. The ARMA model is used to analyze the mechanics of the limb movement of swimming, the Lagrange dynamics model is used to model the

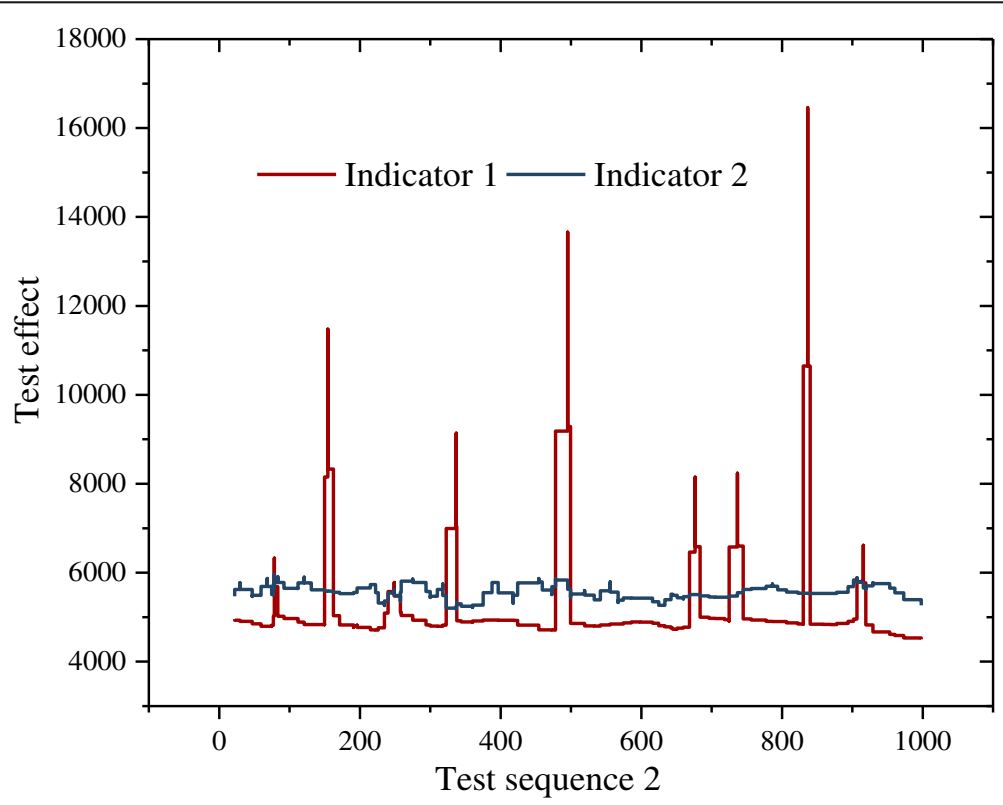

Fig. 4 Network packet loss rate test during swimming dynamics transmission 


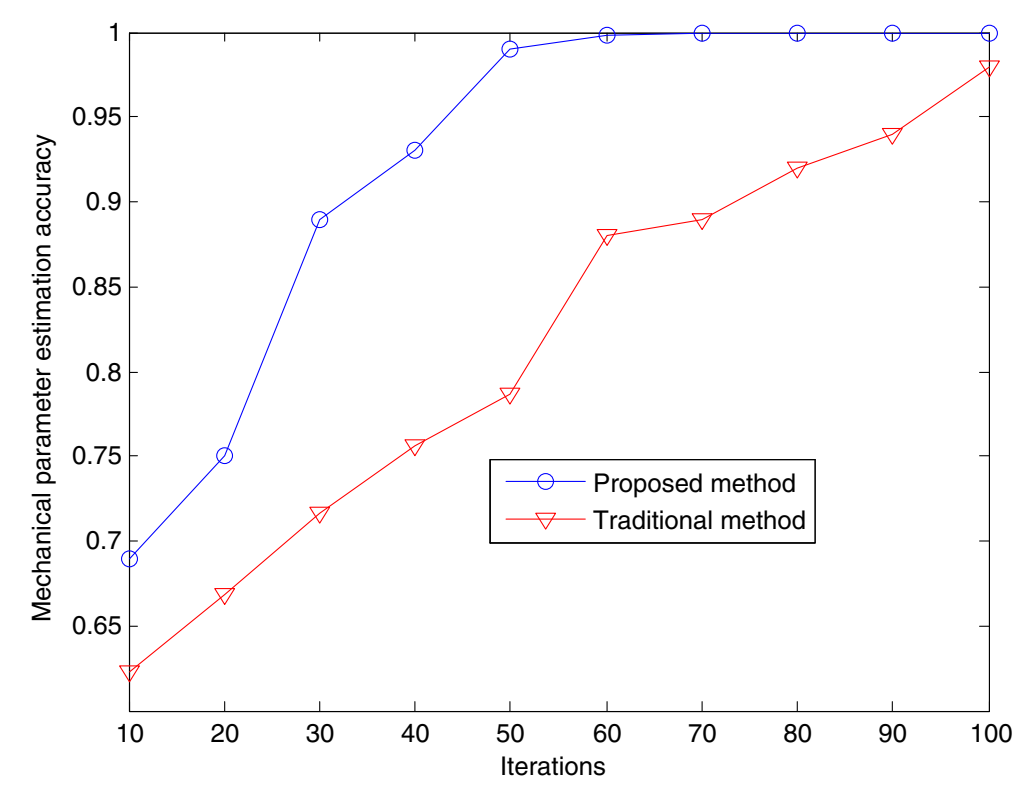

Fig. 5 Accuracy test

characteristics of the mechanics of swimming movement, and the distributed characteristic parameter model of the dynamics of swimming is analyzed in the space of six degrees of freedom. The dynamic distribution space of swimming motion is obtained by using the forward kinematics analysis model, and the inverse kinematics of multiple degrees of freedom is solved by analytic method, and the global and local joint force and force parameters in swimming are estimated. The model of swimming mechanics is realized. The simulation results show that the proposed method is accurate and accurate in estimating the mechanical parameters of swimming movement, and it can effectively guide swimming training. This method has good application value in mechanics modeling and training instruction of swimming.

Abbreviation

ARMA: Agung Rai Museum of Art

Acknowledgements

None

Author's contributions

Zhiwen Xu wrote the entire article. The author read and approved the final manuscript.

Funding

None

Availability of data and materials

The datasets used and/or analyzed during the current study are available from the corresponding author on reasonable request.

Ethics approval and consent to participate

This article does not contain any studies with human participants or animals performed by any of the authors.

Consent for publication

All authors agree to submit this version and claim that no part of this manuscript has been published or submitted elsewhere.

Competing interests

The author declares that he has no conflict of interest. 
Received: 22 April 2020 Accepted: 14 August 2020

Published online: 27 August 2020

\section{References}

1. Q. Gu, L. Yuan, Q.J. Xiong, et al., A comparative study of cost-sensitive learning algorithm based on imbalanced data sets. Microelectron Comput 28(8), 146-149 (2011)

2. Z.X. Li, L.D. Zhao, A SVM classifier for imbalanced datasets based on SMOTEBoost. Syst. Eng. 26(5), 116-119 (2008)

3. W.T. Mao, Y.Y. Tian, J.W. Wang, et al., Granular extreme learning machine for sequential imbalanced data. Control Decision 31(12), 2147-2154 (2016)

4. Q. Gu, L. Yuan, B. Ning, et al., A noval classification algorithm for imbalanced datasets based on hybrid resampling strategy. Comput Eng Sci 34(10), 128-134 (2012)

5. H.P. Guo, J. Zhou, C.A. Wu, M. Fan, K-nearest neighbor classification method for class-imbalanced problem. J Comput Appl 38(4), 955-959 (2018)

6. H.H. Wu, Y. Mu, Z.F. Qu, L.X. Deng, Similarity and nearness relational degree based on panel data. Control Decision 31(3), 555-558 (2016)

7. B. Anqi, D. Aimei, W. Shitong, A dynamic data stream clustering algorithm based on probability and exemplar. J Comput Res Develop 53(5), 1029-1042 (2016)

8. Y.Z. Jiang, F.L. Chung, S.T. Wang, et al., Collaborative fuzzy clustering from multiple weighted views. IEEE Trans Cybernetics 45(4), 688-701 (2015)

9. Z. Fu, X. Sun, Q. Liu, et al., Achieving efficient cloud search services:Multi-keyword ranked search over encrypted cloud data supporting parallel computing. IEICE Trans. Commun. 98(1), 190-200 (2015)

10. W. Shen, L. Wynter, A new one-level convex optimization approach for estimating origin-destination demand. Transp. Res. B Methodol. 46(10), 1535-1555 (2012)

11. C.S. Rao, K.C. Reddy, D.S. Rao, Power control technique for efficient call admission control in advanced wireless networks. Int. J. Comput. Sci. Eng. 4(6), 962-973 (2012)

12. H.Y. Shi, W.L. Wang, N.M. Kwok, et al., Game theory for wireless sensor networks: A survey. Sensors 12(7), 9055-9097 (2012)

13. X. Jingying, N. Xiaoyong, On the reform of college English teaching under the trend of educational informatization. Integr Inform Technol Teach Pract 45(12), 43-45 (2015)

\section{Publisher's Note}

Springer Nature remains neutral with regard to jurisdictional claims in published maps and institutional affiliations.

\section{Submit your manuscript to a SpringerOpen ${ }^{\circ}$ journal and benefit from:}

- Convenient online submission

$\checkmark$ Rigorous peer review

- Open access: articles freely available online

- High visibility within the field

- Retaining the copyright to your article

Submit your next manuscript at $\boldsymbol{s p r i n g e r o p e n . c o m ~}$ 